\title{
CLOSING CEREMONY Basic Questións
}

R.A. Ricci, the President of EPS, in his closing address remarked that the excellent scientific programme and the special EPS-8 sessions raised some basic questions.

The link between fundamental research and technology becomes more heavily debated as science's visibility increases. One can best illustrate this point with an example. There are discusssions going on within EPS itself about the future of optics within its Divisions. Yet the plenary talk by K. Knop demonstrated how a reappraisal of basic principles in optics has led to some intriguing applications. At the same time we use optical technology to investigate basic quantum physics.

Its is therefore perhaps nonsensical to ask if basic research or technical application is the more important as one can exploit science for applied technology and, conversely, technical tools for science, as in the research on quantum crystals reported by A.F. Andreev, and in the study of numerical simulations of physical phenomena described by $R$. Car and M. Parrinello, winners of the 1990 Hewlett-Packard Europhysics Prize. K.A. Müller's talk on high $T_{c}$ superconductors showed that although applications may come before theoretical understanding we do not abandon basic research. On the contrary, without understanding something we are lost and G. 't Hooft even went so far as to seek a "theory of everything" in terms of the primary forces in nature, a remarkable challenge which only fundamental physicists can tackle. This characteristic has been ably demonstrated in unifying descriptions of the Universe of the type proposed by H.A. Bethe in his analysis of exploding supernovae - descriptions that delve into the heart of thermonuclear fusion whose realization as an efficient source of power would have an incredible impact on society. The report of $\mathrm{J}$. Jacquinot on the progress of the European collaboration (JET-MET-ITER) in the magnetic confinement approach to thermonuclear fusion was relevant, although one should not ignore other promising techniques such as inertial confinement with laser or heavy ions. The energy problem and the need for nuclear energy to optimize the environmental issues while sustaining development of a modern society were underlined by H.A. Bethe in the Press Conference.
On the other hand, the basic scientific knowledge related to the properties of our Universe have been reviewed by N.O. Weiss and E. van den Heuvel, and questions concerning fundamental symmetries and primary forces addressed by N.R. Ramsey and J.A. Zakrzewski.

The feedback between fundamental science and technical applications and tools was clearly manifested in the spectacular results obtained at CERN's LEP (700000 $\mathrm{Z}^{\circ}$ events!), as reported by $\mathrm{H}$. Schopper. It was also highlighted by the new ways of exploring the intimate properties of nuclear matter either with electrons (B. Frois) or with heavy ions (R. Stock) and by testing quantum physics with single-atom experiments $(\mathrm{H}$. Walter) and the properties of cold hydrogen atoms using laser studies (T.W. Hänsch).

The vast scope of such a general view of science has also been demonstrated by the impact of physics on microelectronic technology as reviewed by R.P. Kramer ( $4 \mathrm{Mbit}$ in $0.3 \mu \mathrm{m}$ by the year 2000 !) as well as in the contribution of statistical physics to neural network theory (G. Toulouse).

New aspects of current theoretical and experimental work related to the fairly sophisticated behaviour of complex systems (aerogel structure in plasmas, squeezed states of light, mesoscopic systems, fractal geometries by B.H. Smirnov, H.J. Kimble, B.L. Altshuler and L. Pietronero) show once again the vitality of our science and the impressive dichotomy between the simplicity of its basic ideas and the immensity of its applications.

\section{We Need EPS}

EPS has organized specialized conferences in its Divisions for 23 years but during all this time the triennial General Meetings were considered indispensable. They bring physicists, especially younger ones, from many fields together in a general culture. This is only possible if connections between the different branches of physics are preserved within the framework of a society like EPS representing individual physicists, the national societies, organizations and all the different fields of physics through the Divisions.

EPS is therefore a vital ingredient in the physics community so it is perhaps unfortunate that the Society's image as is not as it should be, especially in this

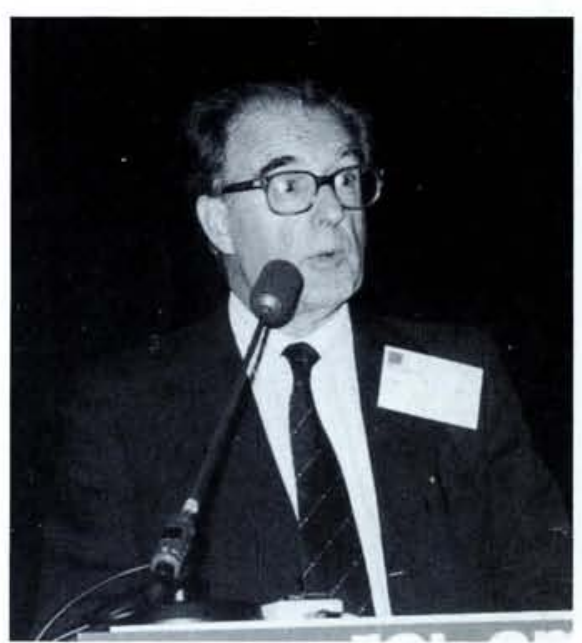

R.A. Ricci, President of EPS speaking at the EPS-8 closing ceremony.

period of increased activity and interchange. EPS is neither a federation, nor a business nor a profit making activity. It represents instead a dream that must be believed in for success and which we have a duty to support. We need only to consult younger colleagues from the east to appreciate why the effort is rewarding.

\section{Professor I. Ursu}

Professor Ricci closed the conference on a slightly unhappy note by reading out the text a telegramme that had been sent on behalf of the participants in reply to I.I. Ursu, the son of Professor I.I. Ursu, a former President of EPS. I.I. Ursu had sent a telegramme to Professor Ricci before the conference explaining that his father, who had been officially invited to attend, would not be able to do so for political reasons. The telegramme from the President read as follows:

To: Ioan I. Ursu

Institute of Atomic Physics

POB MG-6 Bucharest.

\section{Message for your father}

Dear Professor Ursu

The more than 1000 participants in the 8 th General Conference in Amsterdam were deeply concerned about the decision of the Romanian authorities not to permit you to accept my invitation to come to Amsterdam both as a scientist and a Past President of the European Physical Society. We all hope that the importance of your activity in favour of EPS will be recognized and that your personnal situation will improve in order to improve our collaboration. We feel obliged to continue our actions concerning the scientific freedom.

With my best wishes and kind regards on behalf of the European physics community. R.A. Ricci

EPS President 